\title{
Reference Fields Analysis of a Markov Random Field Model to Improve Image Segmentation
}

\author{
E. D. López-Espinoza*1 ${ }^{* 1}$ L. Altamirano-Robles ${ }^{2}$ \\ ${ }^{1}$ Centro de Ciencias de la Atmósfera, \\ Universidad Nacional Autónoma de México, \\ Circuito Exterior $\mathrm{s} / \mathrm{n}$, Ciudad Universitaria, \\ Del. Coyoacan, México, D.F., CP 04510. \\ *danae@atmosfera.unam.mx \\ 2 Instituto Nacional de Astrofísica, \\ Óptica y Electrónica, Luis Enrique Erro No. 1, \\ Sta. Ma. Tonantzintla Puebla, México, CP 72840.
}

\begin{abstract}
In Markov random field (MRF) models, parameters such as internal and external reference fields are used. In this paper, the influence of these parameters in the segmentation quality is analyzed, and it is shown that, for image segmentation, a MRF model with a priori energy function defined by means of non-homogeneous internal and external field has better segmentation quality than a MRF model defined only by a homogeneous internal reference field. An analysis of the MRF models in terms of segmentation quality, computational time and tests of statistical significance is done. Significance tests showed that the segmentations obtained with MRF model defined by means of non-homogeneous reference fields are significant at levels of $85 \%$ and $75 \%$.
\end{abstract}

Keywords: Image segmentation, unsupervised segmentation, Markov random field, non-homogeneous random field.

\section{RESUMEN}

En modelos de Campos Aleatorios de Markov (MRF) se emplean parámetros como el campo de referencia interno y externo. En este artículo, analizamos su influencia en la calidad de la segmentación final, y mostramos que, para segmentación de imágenes, un modelo MRF con una función de energía definida mediante un campo de referencia interno y uno externo no homogéneos, obtiene mejores calidades de segmentación que un modelo MRF definido a través de un solo campo de referencia interno homogéneo. El análisis de los modelos MRF es realizado en términos de la calidad de segmentación, tiempo computacional y pruebas de significancia estadística. Las pruebas de significancia mostraron que los resultados de segmentación obtenidos con el modelo MRF definido a través de campos de referencia no homogéneos son significativos en un nivel del $85 \%$ y $75 \%$.

\section{Introduction}

Image segmentation is a low-level image processing task that aims at partitioning an image into homogeneous regions [1]. With exception of trivial cases, segmentation is not a simple task because the homogeneity concept in regions is difficult to threat in automatic processes. In real images, the objects that can be segmented easily by a human could be erroneously segmented and partitioned in a great amount of small regions by an automatic process.

Image segmentation is important because many high level processing tasks make use of a segmented image, for examples: satellite image classification in remote sensing applications, image understanding and interpretation, object recognition and surface description. In all these tasks, the segmentation results affect severely the results of subsequent processes.

Markov random field [2] is a probabilistic model that has been successfully applied to problems of image segmentation $[3,4]$. MRF models provide a convenient way of modeling spatial contextual information among neighboring pixels. MRF models in computer vision for image segmentation are formulated within the Bayesian framework. The optimal solution of a segmentation problem is defined as the maximum a posteriori (MAP) probability estimate, and it is computed minimizing the posterior energy $p(x \mid y)$. This energy depends on a data likelihood model $p(y \mid x)$ and a priori model $p(x)$ in which contextual constraints are defined. The practical use of MRF models is largely 
ascribed to the equivalence between MRF and Gibbs probability distributions $[2,5]$.

A MRF model called TS-MRF (tree-structured Markov random field) $[6,7,8]$ has been recently used for segmentation $[7,8,9,10]$. TS-MRF is a recursive model with a fast optimization, capable to split highly correlated classes. The differences between TS-MRF model and a FLAT MRF model are 1) TS-MRF uses a binary tree structure to carry out the segmentation and 2) in each tree node a binary FLAT MRF is estimated along with all its associated parameters [6]. In the literature on MRF model based image segmentation $[3,4,7,8,9,10$, $11,12,13,14]$, the Gibbs energy is defined by an auto-model [5], which is a second-order energy function that involves up to pair-site cliques. In this energy only pair-site cliques and a homogeneous internal reference field are used. In $[15,16]$ a Texture Energy Function (TEF) for MRF models used for image segmentation is presented. The TEF consists of a likelihood model with Gaussian distribution $p(y \mid x)$ and a second-order energy function with non-homogeneous internal and external fields obtained by means of the 2-D Wold decomposition [17]. Unlike models in literature, where homogeneity is assumed for mathematical and computational convenience, in this function both fields are considered as non-homogeneous. In this paper, we analyze the importance of nonhomogeneous reference field parameters, as in function TEF, to obtain better image segmentation quality. The analysis is done in terms of segmentation quality, tests of statistical significance and computational time. Significance tests showed that segmentations obtained with the MRF model defined by means of nonhomogeneous reference fields are significant at levels of $85 \%$ and $75 \%$.

\section{Texture Energy Function}

References $[15,16]$ define a prior energy function by means of a non-homogeneous auto-model. The a priori energy function, or Gibbs energy, consists of a second-order energy function with clique potentials defined from texture fields obtained by means of the 2-D Wold decomposition [17]. In 2-D Wold decomposition, the texture image is assumed to be a realization of a 2-D homogeneous random field, and based on Wold decomposition, the texture image is decomposed into a sum of two texture fields: a structural texture field $V$ and a stochastic texture field $W$.

Therefore, the external field of a priori energy function $\alpha_{s}$ is defined by means of a structural texture field $V$ and internal field of a priori energy function $\beta_{s r}$ is defined by means of a stochastic texture field $W$.

Let $\beta_{s r}$ be a function defined as follows:

$$
V_{2}(x)=\left\{\begin{array}{c}
\beta^{s r}=\left|w_{s}-w_{r}\right| \\
0
\end{array} \text { if } s \neq r \text { and } s, r \in \mathrm{C}_{2} \text { and } w_{s}, w_{r} \in W\right.
$$

where $C_{2}$ is the set of pair-site cliques of second order, $w_{s}$ and $w_{r}$ are the gray values at sites $s$ and $r$ for pixels in $W$.

Based on this, the a priori energy is defined by means of reference texture fields $V$ and $W$ as:

$$
U(x)=\sum_{\mathrm{s} \in \mathrm{C} 1} v_{\mathrm{s}} x_{\mathrm{s}}+\sum_{\mathrm{s}, \mathrm{r} \in \mathrm{C} 2} \beta^{s r} x_{\mathrm{s}} x_{\mathrm{r}}
$$


where $x_{s}$ and $x_{r}$ are values at sites $s$ and $r$ for pixels in $X, \beta^{s r}$ is obtained by (1), $v_{s} \in V$ and $C_{1}$ is the set of single-site cliques. On the other hand, the likelihood model is defined as in [7], and finally, the posterior texture energy function TEF is defined as

$$
\begin{gathered}
x_{M A P}=\operatorname{argmin} \sum_{s \in S}\left[\ln 2 \pi^{\frac{B}{2}}\left|\operatorname{Cov}_{k}\right|^{1 / 2}+\frac{1}{2}\left(y_{s}-\mu_{k}\right)^{T}+\left(\operatorname{Cov}_{k}\right)^{-1}\left(y_{s}-\mu_{k}\right)\right. \\
+\ln Z+\frac{1}{Z}\left[\sum_{s \in C_{1}} v_{s} x_{s}+\sum_{s, r \in C_{2}} \beta^{S r} x_{s} x_{r}\right]
\end{gathered}
$$

where $\mu_{k}$ and $\operatorname{Cov}_{k}$ are the mean and the covariance matrices of class $k$ respectively, $y_{s}$ is the gray value in the site $s, B$ is the band number in the image and $Z$ is the partition function. The TEF is integrated within a TS-MRF model and, contrary to models in the literature and to TS-MRF original model, this posterior energy uses both reference fields different from zero $\left(v_{s}=\alpha_{s \neq} 0, \beta^{s r} \neq 0\right)$ and allows non-homogeneous sites.

\section{Analysis of reference fields to improve image segmentation}

We carried out experiments to analyze the influence of parameters such as internal and external reference fields in segmentation quality. Particularly, the performance of the Texture Energy Function (TEF) proposed in $[15,16]$ is evaluated. The TEF uses an a priori energy function with non-homogeneous internal and

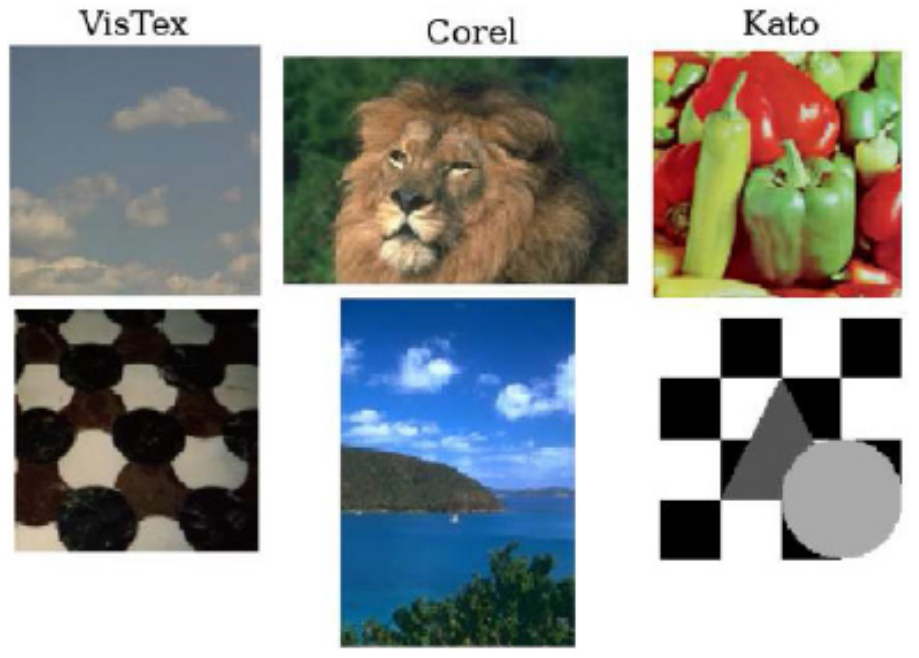

Figure 1. Examples of real and synthetic images. 
external fields obtained by means of the 2-D Wold decomposition [17]. The structural component is defined to be the external field of a priori energy function $\alpha_{s}$ and the stochastic component is defined to be the internal field of a priori energy function $\beta^{\text {sr }}$ (Eq. 1 and 2). The function TEF is integrated within an unsupervised flat MRF model (MRF-FUS), and an unsupervised tree-structured Markov random field model (TS-MRF) in order to evaluate its performance. The function TEF is tested on a variety of synthetic and real images. Real images have been obtained from MIT's VisTex [18] and Corel [19] databases. Real images are segmented manually to obtain the segmentation map and quality is obtained evaluating the percentage of mis-segmented pixels. The synthetic images have been obtained from [20] and synthetic noise is added. Noisy synthetic images were produced by adding 3 kinds of common radiometric noise on remote sensing images [21, 22]: 1) striping (different over all brightness of adjacent lines), 2) drop line (null scan line) and 3) noise (dark and bright points at the background). The first noise is simulated by randomly selecting three lines and setting pixel values to white (255). The second noise is simulated by randomly selecting three lines and setting pixel values to black (0). Finally, the third noise is simulated by adding 0 mean Gaussian noise to the original synthetic image.
Examples of real and synthetic images are shown in Figure 1.

\subsection{Sensitivity of reference fields in the segmentation quality}

First, experiments on synthetic images were carried out in order to analyze how the values of internal and external reference fields are sensitive to initialization. Figure 2 shows how the segmentation quality is influenced by the reference fields $\beta$ and/or $\alpha$. The plot in Figure $2(-\circ-)$ represents segmentation accuracy for the case of a flat MRF model, defined by means of an energy function with an homogeneous internal reference field $\left(\alpha=0\right.$ and $\left.\beta_{\neq} 0\right)$,) whereas the plot in Figure 2 $(-\bullet-)$ represents accuracy for the case of a flat MRF model defined by means of an energy function with homogeneous internal and external fields $\left(\alpha \neq 0\right.$ and $\left.\beta_{\neq 0}\right)$. We can see that a slightly change of initial values of $\alpha$ and $\beta$ may cause that final segmentation accuracy changes. In domains where segmentation depends on very similar object attributes, the problem complexity increases $[15,23]$ and becomes desirable to increase segmentation accuracy in few pixels because on studies on land cover segmentation in remote sensing applications, the final amount of missegmented pixels involves $\mathrm{km}^{2}$ of surface when they are corresponded to the ground. In these

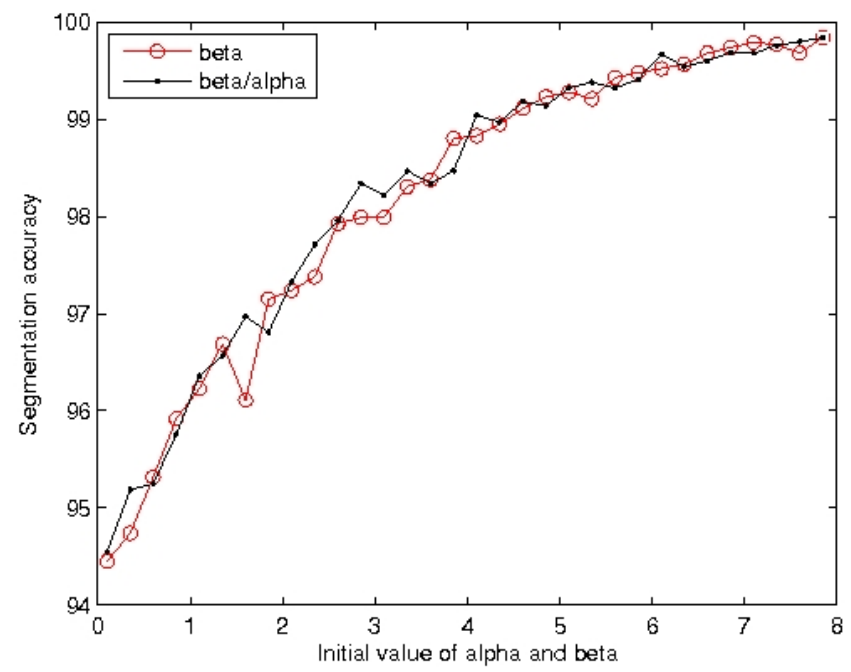

Figure 2. Plot of segmentation accuracy using different values of alpha and beta. 
experiments, we can see from Figure 2 that using an a priori energy function with internal and external fields helps to obtain better accuracy that by using an energy function with only an internal reference field. For example, if we considered $\alpha=0.5$ and $\beta=0.5$, a $95.2 \%$ of segmentation accuracy is reached whereas with only $\beta=0.5$, the percentage falls to $94 \%$. Another example can be seen with $\alpha=1.75$ and $\beta=1.75$ where a $97 \%$ segmentation accuracy is reached and a $96 \%$ with only $\beta=1.75$.

\subsection{Tests of segmentation accuracy}

The application was developed in MATLAB 7.1 to obtain the Wold decomposition and the segmentation using MRF models. The application software has been executed in a computer with an Intel Centrino processor at $1.66 \mathrm{GHz}, 1 \mathrm{~GB}$ RAM in memory and a Linux operating system. To carry out segmentation using the function TEF and any MRF model, first it is necessary to estimate the texture reference fields $V$ and $W$ using Wold decomposition. The Wold algorithm used in the implementation can be found in [24]. The parameters of cut frequency $f_{c}$, evanescent line number $L_{e}$, edge filter and edge threshold threshold ${ }_{e}$, necessary for the decomposition, were manually selected and fitted to each image. An example to obtain the harmonic component of Wold decomposition can be seen in Figure 3 using a cut frequency $f_{c}=11.5$. Figure 4 shows an example to obtain the evanescent component of the same image using 2 evanescent lines $L_{e}$; the edge filter used was Canny and edge threshold threshold $=0.03$. The structural component $W$ is estimated by summing the harmonic and evanescent component as shown in Figure 5. Finally, the stochastic component is obtained by the remaining image (see Figure 6).

Once the Wold decomposition has been carried out, the segmentation can be obtained using the function TEF and the TS-MRF model (see Figure 7).

The Correct Segmentation Accuracy (CSA) is estimated using Equation (4).

$$
\text { CSA }=\frac{\text { Total number of correct segmented pixels }}{\text { Total number of pixels at the image }}
$$

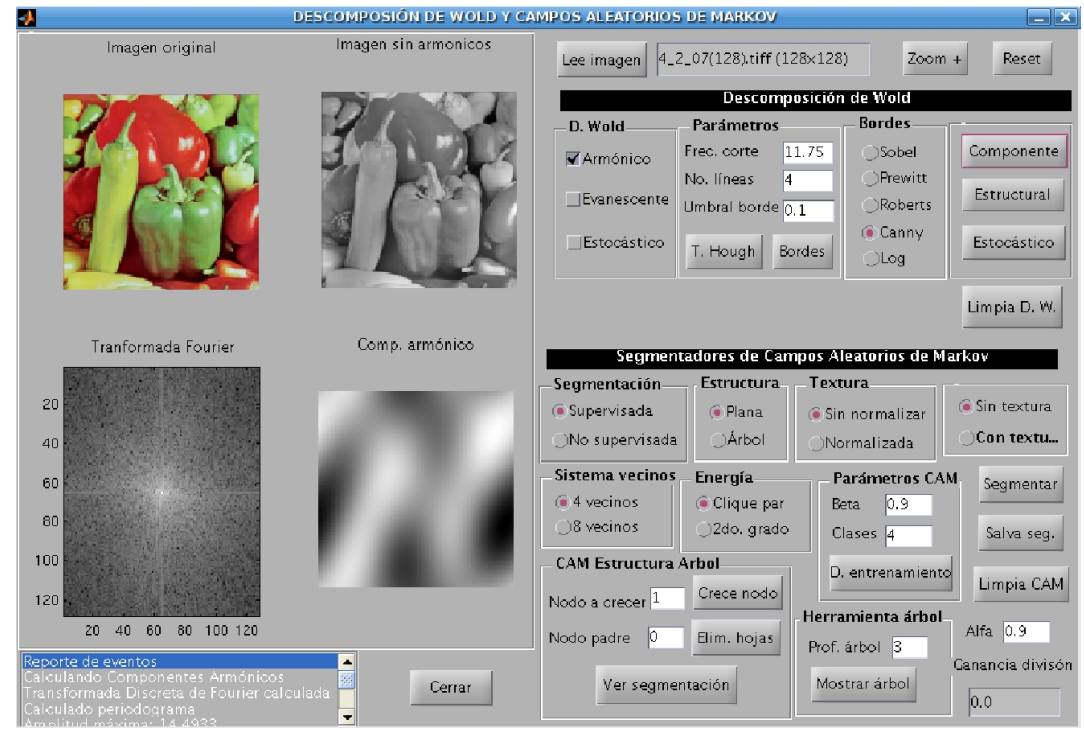

Figure 3. Obtaining harmonic component. 


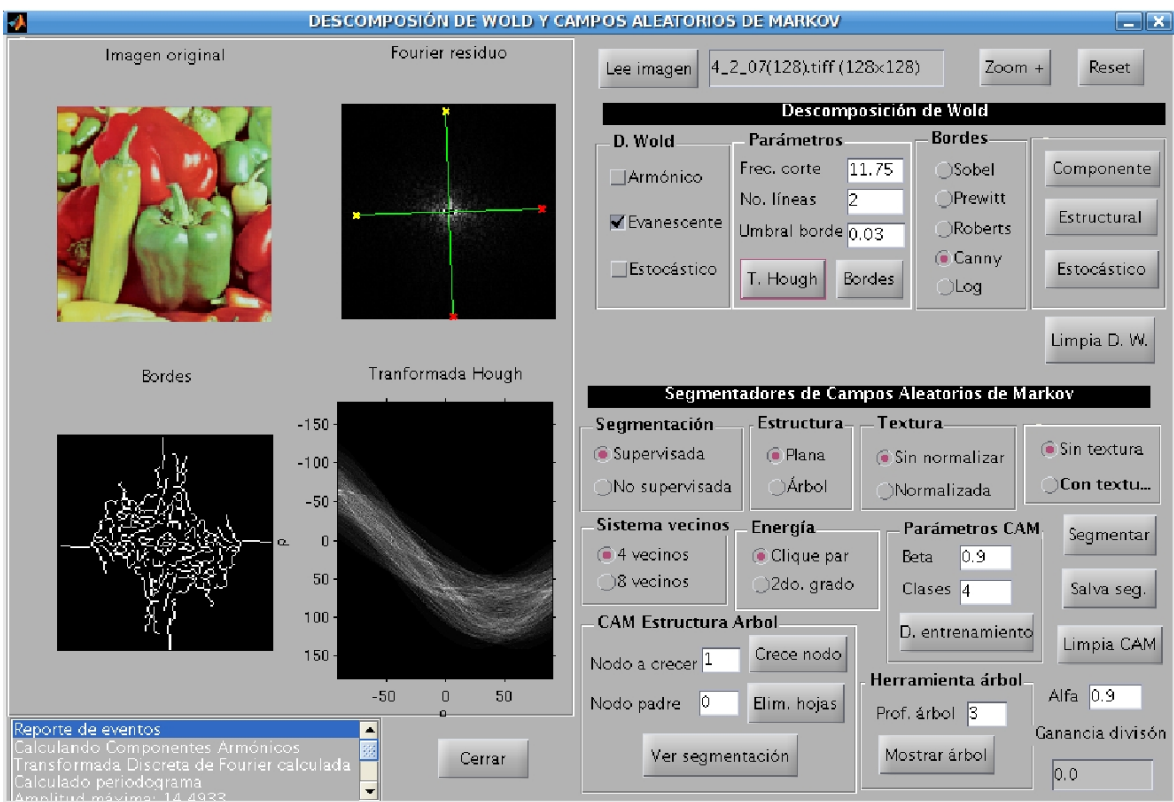

Figure 4. Obtaining evanescent component.

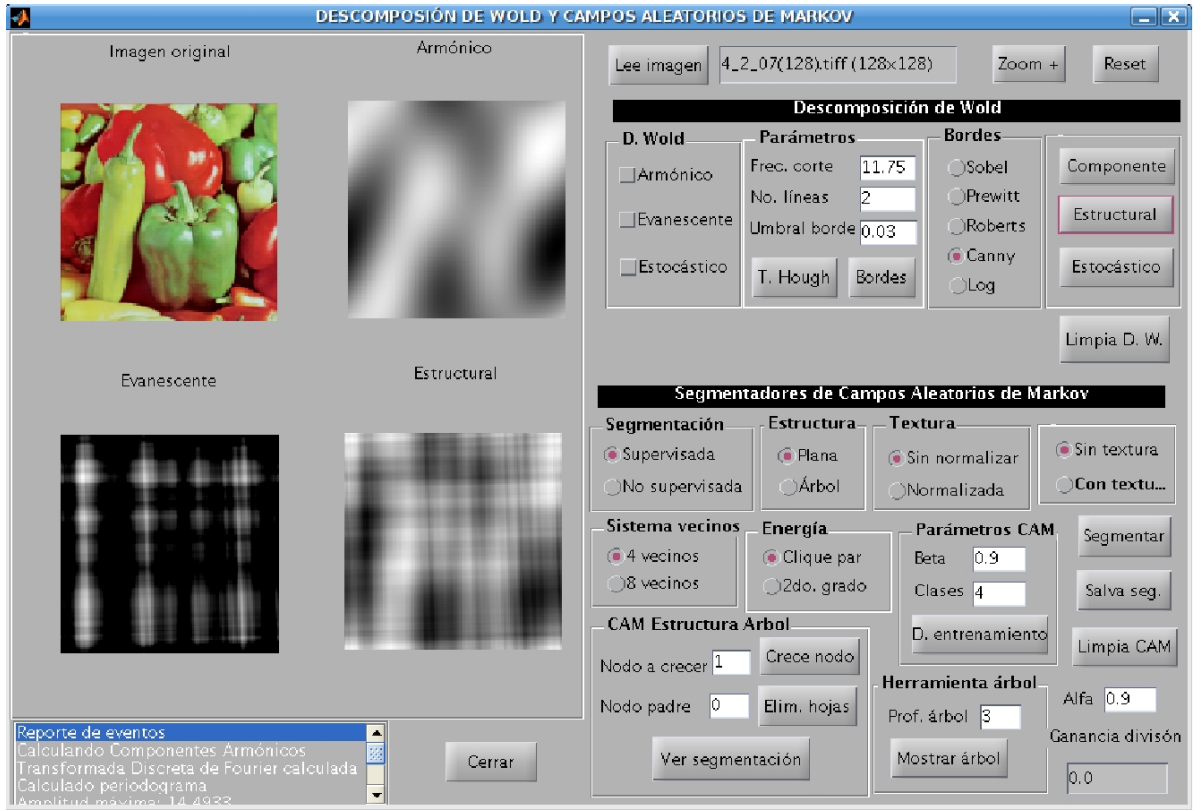

Figure 5. Obtaining structural component $W$. 


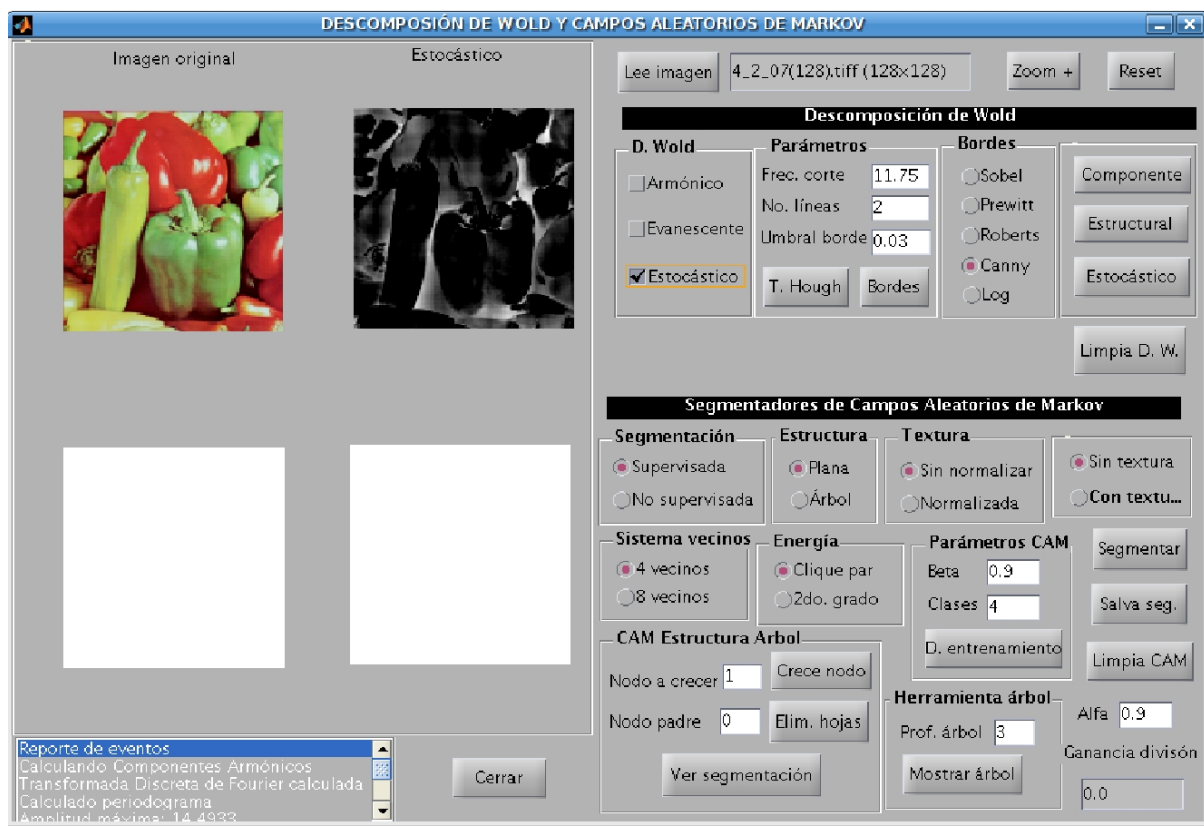

Figure 6. Obtaining stochastic component $V$.

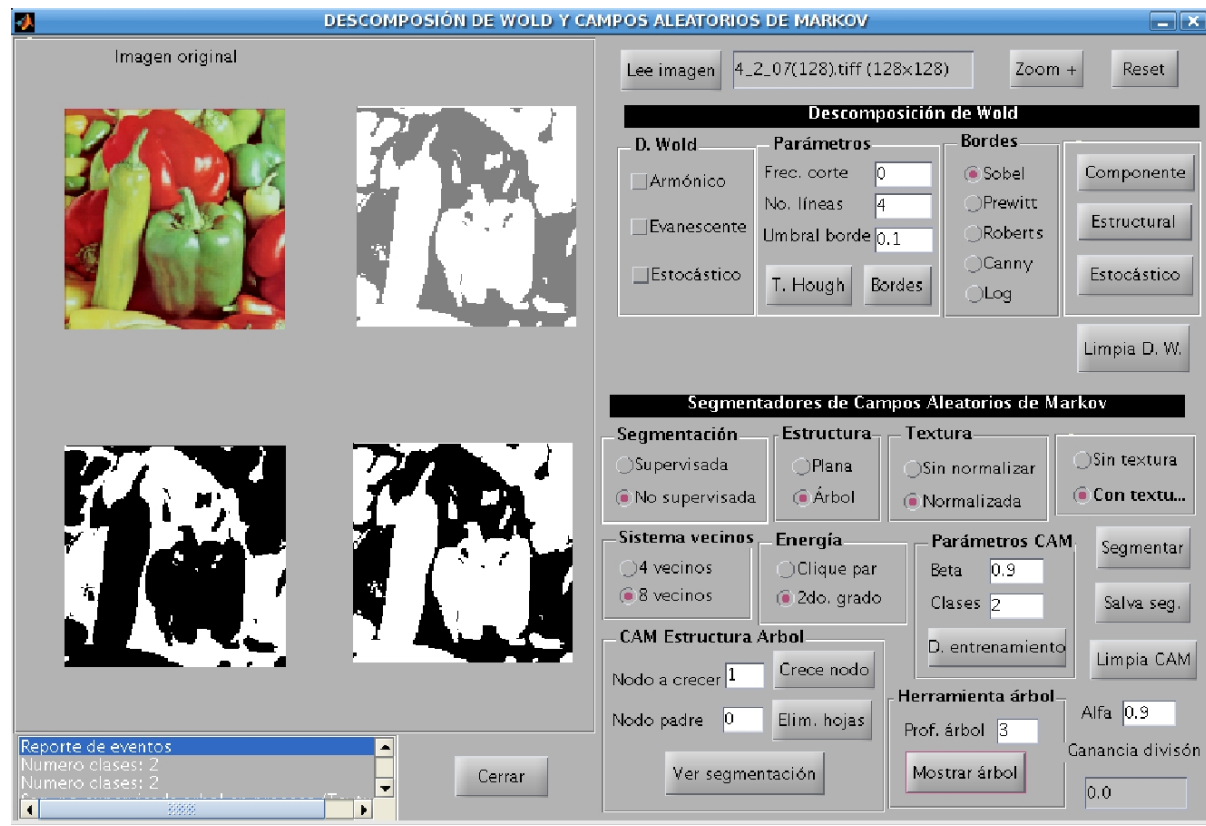

Figure 7 Segmentation using TS-MRF/TEF. 
The correct segmentation accuracy obtained with Equation (4) for synthetic images using MRF models is presented in Tables 1 and 2 . We can see that, for the TS-MRF/TEF model, the correct segmentation accuracy decreases as the image is contaminated by a greater amount of noise. Something similar happens with the TS-MRF original model. In the six experiments, the TSMRF/TEF model obtained the best results.

Figure 8 shows a visual comparison between the segmentation obtained with MRF-FUS and the segmentation obtained with TS-MRF/TEF. In this image, 2 classes were defined: airplanes and sky. We can see that the image is better segmented using the TS-MRF/TEF model.

Figure 9 shows a visual comparison for two images between the segmentation obtained with MRF-FUS, MRF-FUS/TEF and TS-MRF/TEF. The segmentation is improved, when the function TEF is introduced to an MRF-FUS model (see examples in Figure $9 a$ and 9b). Figure 9c shows how a FLAT segmentation with function TEF is improved when both an MRF model not FLAT (i.e. a model based in a binary tree structure) and the function TEF are introduced.

Figure 10 shows a visual comparison for a remote sensing image. In this case, segmentations obtained with TS-MRF (Figure 10a) and TSMRF/TEF models (Figure 10b) are compared. Table 3 shows other correct segmentation accuracies for real images. We can see that the segmentation accuracy is improved when the function TEF is introduced.

\begin{tabular}{|l|c|c|}
\hline \multicolumn{1}{|c|}{ Image } & TS-MRF & TS-MRF/TEF \\
\hline 13dB & 91.64 & $\mathbf{9 2 . 0 2}$ \\
5dB & 59.2 & $\mathbf{7 4 . 4 4}$ \\
3dB & 64.19 & $\mathbf{6 4 . 2 9}$ \\
\end{tabular}

Table 1. Segmentation accuracy using TS-MRF and TS-MRF/TEF models for Gaussian noise.

\begin{tabular}{|l|c|c|}
\hline \multicolumn{1}{|c|}{ Image } & TS-MRF & TS-MRF/TEF \\
\hline \hline 13dB + Striping + Drop line & $\mathbf{8 9 . 3 2}$ & 89 \\
5dB + Striping + Drop line & 65.7 & $\mathbf{6 5 . 8 2}$ \\
3dB + Striping + Drop line & 59.39 & $\mathbf{5 9 . 5 1}$ \\
& & \\
\hline
\end{tabular}

Table 2. Segmentation accuracy using TS-MRF and TS-MRF/TEF models for Striping, Drop line and Gaussian noise.

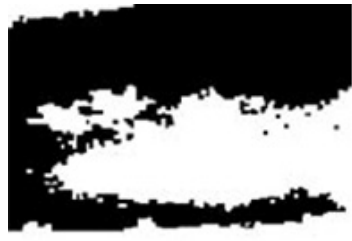

$\mathbf{a}$

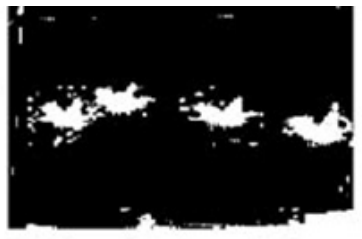

b

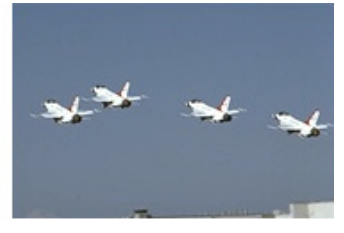

c

Figure 8. Segmentation using a) MRF-FUS, b) TS-MRF/TEF and c) original image. 


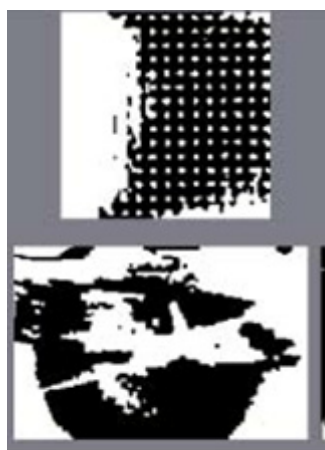

$\mathbf{a}$

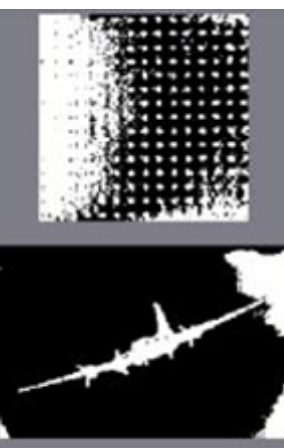

b

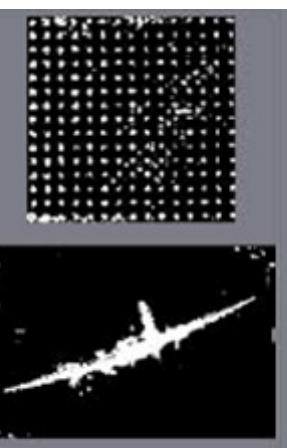

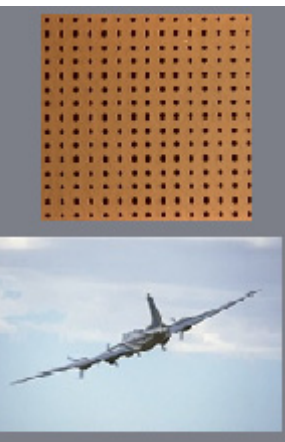

d

Figure 9. Segmentation using a) MRF-FUS, b) MRF-FUS/TEF, c) TS-MRF/TEF and d) original image.
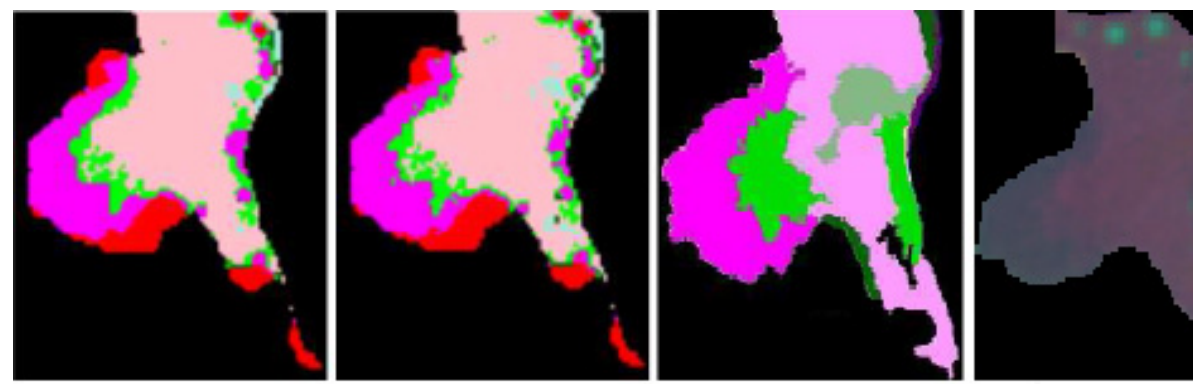

Figure 10. Segmentation using a) TS-MRF, b) TS-MRF/TEF, c) real segmentation obtained by means of field studies and d) original image.

\begin{tabular}{|l|c|c|c|}
\hline \multicolumn{1}{|c|}{ Image } & MRF-FUS & TS-MRF & TS-MRF/TEF \\
\hline \hline Image 6 & 52.43 & 64.51 & $\mathbf{6 4 . 7 4}$ \\
Image 8 & 87.91 & $\mathbf{9 3 . 7 3}$ & 93.51 \\
Remote sensing & -- & 57.05 & $\mathbf{5 8 . 3 9}$ \\
image & & & \\
\hline
\end{tabular}

Table 3. Segmentation accuracy using MRF-FUS, TS-MRF and TS-MRF/TEF models for real images. 


\subsection{Tests of statistical significance}

In order to find the significance level of the results, first the MRF-FUS model is compared against the TS-MRF/TEF model. Afterwards, we compared the original model TS-MRF against the TS-MRF/TEF model. To estimate the significance level of different observations in each sample with homogeneous variance, the following equation was used:

$$
t=\frac{X_{A}-X_{B}}{\sqrt{\frac{\gamma_{A B}^{2}}{N A}+\frac{\gamma_{A B}^{2}}{N B}}}
$$

where $X_{A}$ and $X_{B}$ denote the average of samples $A$ and $B$ respectively, $N A$ and $N B$ are the number of observations in $A$ and $B$, respectively, and $V_{A B}^{2}$ is the variance of $A B$ defined as

$$
\gamma_{A B}^{2}=\frac{(N A-1) \gamma_{A}^{2}+(N B-1) \gamma_{B}^{2}}{(N A-1)+(N B-1)}
$$

where $\mathrm{Y}_{\mathrm{A}}^{2}$ and $\mathrm{Y}_{\mathrm{B}}^{2}$ are the variances of samples $A$ and $B$, respectively.

First, the significance level for MRF-FUS and TSMRF/TEF models is obtained. Taking into account the segmentation accuracy of the previous section, and considering the TS-MRF/TEF model as sample $A$ and the MRF-FUS model as sample $B$, we obtained the statistics for each sample (see Table 4). In order to evaluate homogeneity of variances, we divided the largest variance (sample $B$ ) by the smallest variance (sample $A$ ) to give an $F$-ratio. In this case a value of 1.11 is computed. Now, searching in an $F$-Table using the numerator degrees of freedom $(N B-1)$, and the denominator degrees of freedom $(N A-1)$, a value of 3.074 is found. Because the computed value is less than the tabled value, we can assume homogeneity of variances. Using Equation 4 in this experiment, a value of 1.21018 is computed. Searching in a ttable with $(N A-1)+(N B-1)$ degrees of freedom, a value of 1.0880 is obtained at a significance level of $85 \%$. We can see that this value is less than the tabled value $t$; therefore, we can assume that the segmentation differences were statistically significant.

\begin{tabular}{|c|c|c|}
\hline & Sample A & Sample B \\
\hline \hline $\mathrm{N}$ & 8 & 4 \\
\hline$\sum x_{i}$ & 679.85 & 289.47 \\
\hline Average X & 84.9812 & 72.3675 \\
\hline$\sum x_{i}^{2}$ & 59388.7655 & 21716.9215 \\
\hline$\gamma^{2}$ & 230.609 & 256.2338 \\
\hline $\begin{array}{l}\text { Standard } \\
\text { deviation }\end{array}$ & 15.1858 & 16.0073 \\
\hline
\end{tabular}

Table 4. Statistics for TS-MRF/TEF and MRF-FUS models.

On the other hand, in Table 5, statistics to obtain the significance level between TS-MRF and TSMRF/TEF models are shown. In this test, the TSMRF model is considered as sample $A$ and the TSMRF/TEF model is considered as sample $B$. The computed value $F$ is 1.15 , searching in the $F$-Table using as numerator 10 degrees of freedom, and as denominator 13 degrees of freedom, we found a value of 2.138. Because the computed value is less than the tabled value, we can assume homogeneity of variances. Using Equation 4, a value of 0.79104 is computed. Searching in a $t$ Table with 23 degrees of freedom, a value of 0.6853 is obtained at a significance level of $75 \%$. This value is less than the tabled value $t$; therefore, we can assume that the segmentation differences were statistically significant

\begin{tabular}{|c|c|c|}
\hline & Sample A & Sample B \\
\hline \hline $\mathrm{N}$ & 11 & 14 \\
\hline$\sum x_{i}$ & 830.01 & 1125.37 \\
\hline Average X & 75.4555 & 80.3836 \\
\hline$\sum x_{i}^{2}$ & 65210.6731 & 93378.2385 \\
\hline$\gamma^{2}$ & 258.1891 & 224.383 \\
\hline $\begin{array}{c}\text { Standard } \\
\text { deviation }\end{array}$ & 16.0683 & 14.9794 \\
\hline
\end{tabular}

Table 5: Statistics for TS-MRF/TEF and TS-MRF models. 


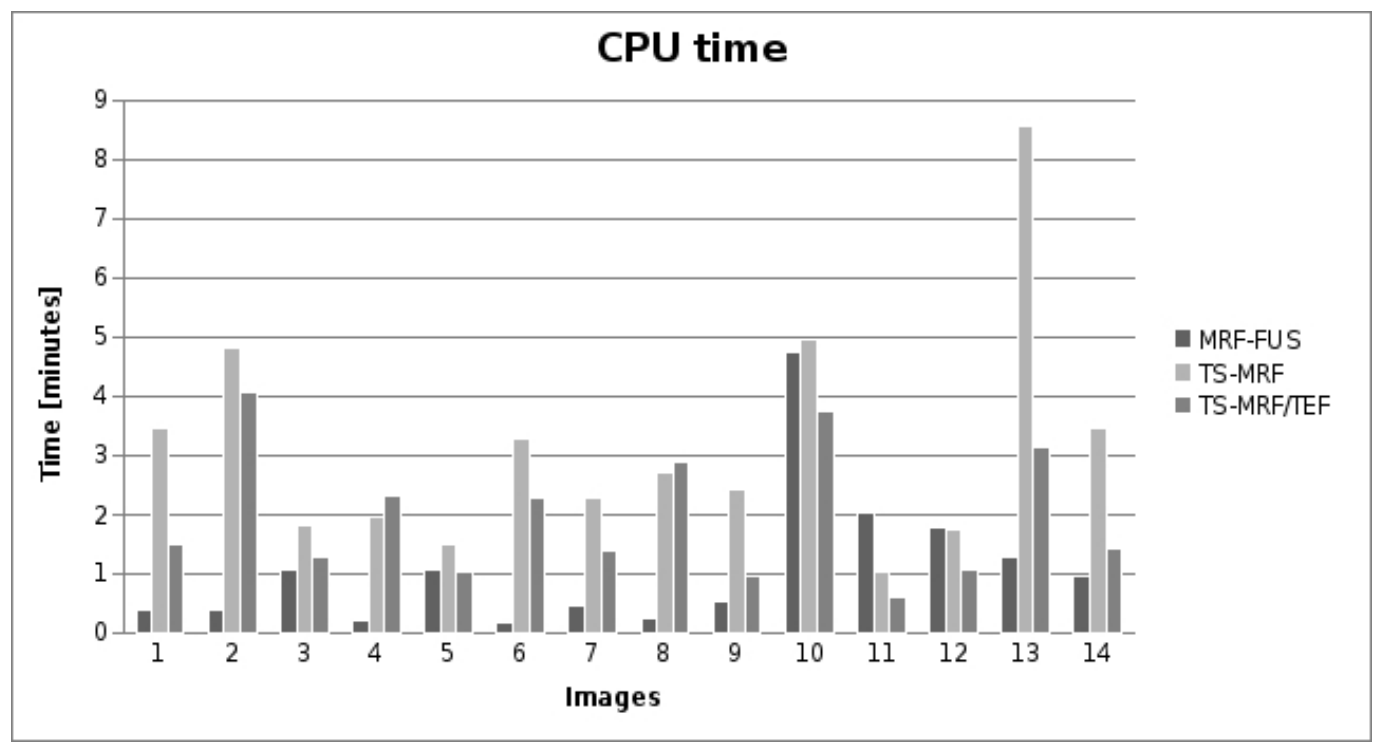

Figure 11. CPU time.

\subsection{Computational time analysis}

Figure 11 shows the CPU time for some test images. In general, the CPU time for the MRF-FUS model is less than the CPU time for the TS-MRF and TS-MRF/TEF models due to the fact that in these models the reference field parameter estimations are not done, and the internal reference field $\beta$ is set to 0.9 because this value has been found to provide satisfactory results in the experiments. On the other hand, the CPU time for the TS-MRF/TEF model is less than the CPU time for the TS-MRF model in 12 of 14 segmentations. In both models, parameter estimations are done. TS-MRF uses PML (Pseudo-likelihood) [2, 25] whereas TS-MRF/TEF uses Wold decomposition [17]. The nonhomogeneity of reference fields did not affect the CPU time of the model. Average CPU time for segmentations using an MRF-FUS model is one minute, for the TS-MRF/TEF model is two minutes and for the TS-MRF is three minutes.

\section{Conclusion}

This paper has analyzed the influence of parameters as internal and external reference fields of MRF models in the segmentation quality. Experiments are done on synthetic images to show that an a priori energy function with internal and external fields, as in texture energy function TEF, helps to obtain better segmentation accuracy than an energy function with only an internal reference field. Experiments with synthetic and real images show that the model that uses the function TEF (TS-MRF/TEF) improves the segmentation quality, and statistical significance tests showed that the segmentations obtained with this model are significant at the $85 \%$ level in comparison with a flat MRF model, and are significant at the $75 \%$ level in comparison with a TS-MRF model. In conclusion, we can say that differences between the obtained segmentations are caused by the introduction of the function TEF. In addition, the CPU time for TS-MRF/TEF model is comparable with the CPU time for TS-MRF. 


\section{References}

[1] Akram A. Moustafa and Z. A. Alqadi, Reconstructed color image segmentation, Proceedings of the World Congress on Engineering and Computer Science WCECS'09, ISBN:978-988-18210-2-7, Vol II:1282-1285, October 20-22, 2009, San Francisco, USA.

[2] S. Z. Li., Markov Random Field Modeling in Computer Vision, Chapter 1 http://www.cbsr.ia.ac.cn/users/szli/MRF_Book/book.html, (accessed March 2010).

[3] Z. Kato and T-Chuen Pong, A Markov random field image segmentation model for color textured images, Image and Vision Computing, 24:1103--1114, January 2006.

[4] Z. Kato, T-Chuen Pong, and J. Chung-M. Lee. Color image segmentation and parameter estimation in a Markovian framework. Pattern Recognition Letters, 22:309--321,2001.

[5] S. Geman and D. Geman, Stochastic relaxation, Gibbs distributions, and the bayesian restoration of images, IEEE Transactions on Pattern Analysis and Machine Intelligence, 6:721--741,1984.

[6] G.Poggio and A. R. Averbuch, Image segmentation by tree-structured Markov random field, IEEE Signal Processing Lett., pages 155--157, July 1999.

[7] C. D'Elia, G. Poggi, and G. Scarpa, A tree-structured Markov random field model for Bayesian image segmentation, IEEE Trans. on Image Proc., 12 (10):1259--1273, October 2003.

[8] G. Poggi, G. Scarpa, and J. Zerubia, Supervised segmentation of remote-sensing images based on a tree-structured MRF model, IEEE Transaction on Geoscience and Remote Sensing, 43(8):1901--1911, August 2005.

[9] G. Cuozzo, Dapos, C. Elia, and V. Puzzolo, A method based on tree-structured Markov random field for forest area classification, IEEE International Geoscience and Remote Sensing Symposium, 2:2352--2354, 2004.

[10] B. Aiazzi, L. Alparone, S. Baronti, G. Cuozzo, C. D'Elia, and G. Schirinzi. SAR image segmentation through information theoretic heterogeneity feature and tree-structured Markov random fields. Proc. IEEE International Geoscience and Remote Sensing Symposium, 4:2803--2806, 2005.
[11] X. Zheng F. Li, J. Peng, Object-based and semantic image segmentation using MRF. EURASIP Journal on Applied Signal Processing, 6:883--840, 2004.

[12] M. Haindl G. Scarpa, Unsupervised texture segmentation by spectral-spatial-independent clustering, 18th International Conference on Pattern Recognition ICPR, 2:151--154, 2006.

[13] I. Kovtun. Texture segmentation of images on the basis of Markov random fields, Tech. rep.: TUD-FI03, 05 2003, Department of Image Processing and Recognition, International Research and Training Centre for Information Technologies and Systems, Dresden Technical University, Kiev, Ukraine.

[14] Z. Kato, Bayesian color image segmentation using Reversible Jump Markov Chain Monte Carlo, Research Report PNA-R9902, Centrum voor Wiskunde en Informatica, 1999.

[15] E. D. López-Espinoza and L. Altamirano-Robles, A method based on tree-structured Markov random field and a texture energy function for classification of remote sensing images, Workshop on Computational Advances in Processing Remote Sensing Imagery CASI2008, part of the 5th International Conference CCE08, IEEE Catalog Number: CFP08827 -CDR, ISBN: 978-1-42442499-3.

[16] E. D. López-Espinoza and L. Altamirano-Robles, Segmentación Markoviana usando modelos de textura, Reporte técnico No. CCC-09-002, Coordinación de Ciencias Computacionales, INAOE, Mayo 2009.

[17] B. Porat J. M. Francos, A.Z. Meiri. A unified texture model based on a 2-d wold like decomposition. IEEE Transactions on Signal Processing, 41:2665--2678, 1993.

[18] R. Picard, C. Graczyk, S. Mann, J. Wachman, L. Picard, and L. Campbell, MIT VisTex texture database, Vision and Modeling Group of the MIT Media Laboratory, Cambridge, Massachusetts, http://vismod.media.mit.edu/vismod/imagery/VisionTextu re/vistex.html (accessed March 2010).

[19] P. Carbonetto, Corel photography set, Department of Human Genetics University of Chicago, http://www.cs.ubc.ca/ pcarbo/\#data, (accessed March 2010).

[20] M. Berthod, Z. Kato, S. Yu, and Josiane Zerubia, Bayesian Image Classification Using Markov Random Fields. Image and Vision Computing, 14:285--295, 1996. 
[21] S. Mohammad Shahrokhy, Visual and statistical quality assessment and improvement of remotely sensed images, In Geo-Imagery Bridging Continents, XXth ISPRS Congress, Vol. XXXV, ISSN 1682-1750, Istanbul, Turkey p. 104--108, July 2004.

[22] J. Torres and S. O. Infante, Wavelet analysis for the elimination of striping noise in satellite images, Society of Photo-Optical Instrumentation Engineers, 40:1309-1314, 2001.

[23] B. Tsai, T. Kojima, N. Kosaka, T. Akiyama, Forest type classification using data fusion of multispectral and panchromatic high-resolution satellite imageries, Geoscience and Remote Sensing Symposium, IGARSS apos;05 Proceedings, IEEE International, 4:12980-2983, 2005.

[24] E. D. López-Espinoza and L. Altamirano-Robles, Deterministic component of 2-d Wold decomposition for geometry and texture descriptors discovery, Lecture Notes in Computer Science, Progress in Pattern Recognition, Image Analysis and Applications, 4756/2008:241--250, 2007.

[25] G. Winkler, Image Analysis, Random Fields and Markov Chain Monte Carlo Methods, Springer-Verlag, Second edition, New York, Part 6:268, 2006. 


\section{Authors' Biographies}

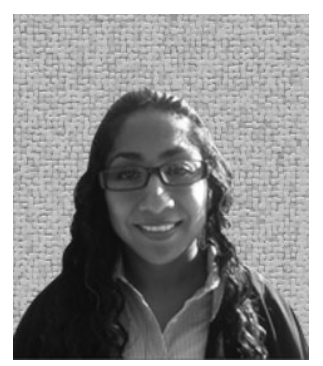

\section{Erika Danaé LÓPEZ-ESPINOZA}

She received the $\mathrm{BSc}$ degree in computer science from Benemérita Universidad Autónoma de Puebla (BUAP) in 2002, and the MSc and PhD degree in computer science from Instituto Nacional de Astrofísica, Óptica y Electrónica (INAOE), in 2004 and 2009, respectively. From 2004 to 2005, she was part of the Computer Vision Laboratory team at INAOE, and from 2008 to 2009 she worked at the Mexico City's Virtual Center on Climate Change. Since March 2010, she is doing postdoctoral research at Centro de Ciencias de la Atmósfera, UNAM. Her research interests are pattern recognition, computer vision, remote sensing and numerical weather prediction models.

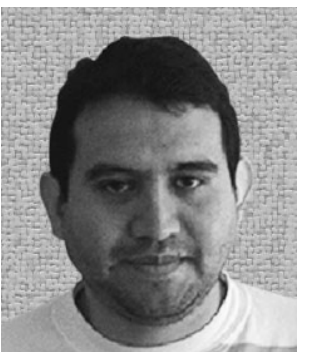

\section{Leopoldo ALTAMIRANO-ROBLES}

He received the BSc degree in computer science from BUAP in 1985, the MSc degree in electric engineering from Centro de Investigación y de Estudios Avanzados (CINVESTAV) in 1991 and the PhD degree from Technische Universität München, Germany in 1996. Since 1999, he is member of the Sistema Nacional de Investigadores (National System of Researchers), level 1. He is professor at the Computer Science Department and head of the Computer Vision Laboratory at INAOE. His research lies on computer vision, sensor fusion and industrial applications. 